\title{
Positive Lyapunov Exponents for a Class of Ergodic Schrödinger Operators
}

\section{Steve Surace}

Drew University, Department of Mathematics, Madison, New Jersey 07940, USA

Received: 1 June 1993/in revised form: 10 August 1993

Abstract: We present a simple method to estimate the Lyapunov exponent $\gamma(E)$ for the system

$$
-\left(\psi_{j+1}+\psi_{j-1}\right)+v_{j}(\omega) \psi_{j}=E \psi_{j},
$$

where $\left\{v_{j}(\omega)\right\}_{\omega \in \Omega}$ is an ergodic family of potentials defined for $j \in \mathbb{Z}$. We assume that there is a constant $\zeta>2$ and large positive integers $l, L$ such that for almost every $\omega$ and every $E$ there is an infinite sequence of disjoint intervals $J_{n} \subset \mathbb{Z}$ with the following properties:

1) The length of each interval is larger than $2 l$.

2) The distance between any two adjacent intervals is less than $L$.

3) $\left|v_{j}(\omega)-E\right| \geqq \zeta$ for $j \in \bigcup_{n} J_{n}$.

Under these conditions we prove that

$$
\text { meas }\{E: \gamma(E)=0\} \leqq B e^{-\beta l / 6} .
$$

where $\beta$ and $B$ are positive constants and "meas" refers to Lebesgue measure.

\section{Introduction}

In this paper we obtain the following result:

Theorem. Consider the finite difference Schrödinger equation

$$
-\left(\psi_{j+1}+\psi_{j-1}\right)+v_{j}(\omega) \psi_{j}=E \psi_{j} .
$$

where $\left\{v_{j}(\omega)\right\}_{\omega \in \Omega}$ is an ergodic family of potentials defined for $j \in \mathbb{Z}$. Let $\gamma(E)$ be the Lyapunov exponent for (1) and let $A \equiv\{E \in \mathbb{R}: \gamma(E)=0\}$. Suppose that there is a constant $\zeta>2$ and large positive integers $l, L$ such that for almost every $\omega$ and every $E$ there is an infinite sequence of disjoint intervals

$$
J_{n}=\left[a_{n}-l_{n}, a_{n}+l_{n}\right] \subset \mathbb{Z}
$$


$(n \in \mathbb{Z})$ with the following properties:
(i) $a_{n}<a_{n+1}$,
(ii) $l_{n} \geqq l$,
(iii) $a_{n+1}-a_{n} \leqq L$,
(iv) $\left|v_{j}(\omega)-E\right| \geqq \zeta$ for $j \in \bigcup_{n} J_{n}$.

Then

$$
\operatorname{meas}(A) \leqq B e^{-\beta l / 6},
$$

where $\beta$ and $B$ are positive constants.

Remarks.

1. The constant $\beta$ depends on $\zeta$ while $\mathrm{B}$ depends on $\zeta$ and on the ratio $l / L$.

2. The set $A$ in the above inequality can be replaced by

$$
\{E \in \mathbb{R}: \gamma(E) \leqq b\},
$$

where $b$ depends on $\zeta$ and on the ratio $l / L$.

3. If the hypothesis of the theorem is true only for those values of $E$ which belong to some measurable subset $S \subset \mathbb{R}$, then it follows that

$$
\text { meas }\{A \cap S) \leqq B e^{-\beta l / 6} .
$$

4. The size of $l, L$ needed to make the conclusion interesting depends on the size of $1 /(\zeta-2)$.

Our result yields the following corollaries:

Corollary 1. Let $f(\vartheta)$ be continuous and periodic of period one. Suppose that there exists a constant $\zeta>2$ such that for each value of $E$ there exists a point $\vartheta^{*}$, where

$$
\left|f\left(\vartheta^{*}\right)-E\right|>\zeta \text {. }
$$

Consider system (1) with the potential

$$
v_{j}(\vartheta)=f(\alpha j+\vartheta)
$$

where $\alpha$ is a small irrational number. Then the Lyapunov exponent is positive except for those values of $E$ which belong to a set of measure $\cong e^{- \text {Const } / \alpha}$.

There is a special case of our theorem which applies to the perturbed almost Mathieu operator:

Corollary 2. Consider system (1) with the potential

$$
v_{j}(\vartheta)=\lambda \cos 2 \pi(\alpha j+\vartheta)+\varepsilon f(\alpha j+\vartheta) .
$$

We assume that

(i') $\lambda>2$,

(ii') $f$ is a bounded measurable function of period 1 ,

(iii') $\varepsilon\|f\|_{\infty}<(\lambda-2) / 2$,

(iv') $\alpha$ is a small irrational number.

Under conditions ( $\left.\mathrm{i}^{\prime}\right)$-(iv') it follows that the Lyapunov exponent for the system associated with (2) is positive except for those values of $E$ which belong to a set of measure $\cong e^{- \text {Const } / \alpha}$. 
Remark. Corollaries 1 and 2 may be adapted to the case where $\alpha$ is a small non-zero rational number.

Recently there have been several results along these lines. Systems such as (1), (2) come up in the study of quantum mechanics, solid state physics and dynamical systems. We now briefly mention some of the latest results in this area.

Consider the system

$$
-\left(\psi_{j+1}+\psi_{j-1}\right)+\lambda v_{j}(\vartheta) \psi_{j}=E \psi_{j}
$$

where $v_{j}(\vartheta)$ is an ergodic potential defined for $j \in \mathbb{Z}$. Then for large $\lambda$ and suitable conditions on $v_{j}$ (see [1]), it is known that the measure of the set of energy $E$ where the Lyapunov exponent is zero is exponentially small in $\lambda$. Our theorem improves upon the result in [1] because we don't need to assume that $\lambda$ is very large.

There is another recent result by Herman [2] which applies to the system

$$
-\left(\psi_{j+1}+\psi_{j-1}\right)+\lambda \cos 2 \pi(\alpha j+\vartheta) \psi_{j}=E \psi_{j},
$$

where $\alpha$ is irrational. Herman obtains the lower bound on the Lyapunov exponent $\gamma(E) \geqq \log (\lambda / 2)$. Therefore if $\lambda>2$, then the Lyapunov exponent is positive. One drawback with the method used by Herman is that the proof breaks down under perturbations of the cosine potential. Our result is valid for potentials (2) which are "close" to the cosine potential.

Sorets and Spencer [3] discovered an extension of Herman's result. They get estimates on the Lyapunov exponent for the system

$$
-\left(\psi_{j+1}+\psi_{j-1}\right)+[\lambda \cos 2 \pi(\alpha j \pm \vartheta)+\varepsilon f(\alpha j+\vartheta)] \psi_{j}=E \psi_{j},
$$

where $f$ is a real analytic function of period one and $\alpha$ is irrational. In particular they show that for large $\lambda$ and small $\varepsilon$ the Lyapunov exponent is positive for every energy $E$ in the spectrum and for almost every $\vartheta$. Their result successfully deals with perturbations of the cosine potential, but in their proof they must assume that the perturbation function $f$ is analytic. Our result improves upon [3] because we only need to assume that $f$ is bounded and not necessarily analytic. In addition our proof does not require $\lambda$ to be very large.

The results that we mentioned above as well as the result in this paper are obtained using "soft" analysis techniques as opposed to the difficult multi-scale perturbation analysis which is usually used in problems of this type $[4,5,8]$. ideas.

Before we give the proof of our result we shall give a brief outline of the main

\section{Definitions, Notation and an Outline of the Proof}

Definitions and Notation. We wish to study system (1) with conditions (i-iv). We shall frequently write this system as the matrix equation

$$
(-\Delta+V) \psi=E \psi,
$$

where $\Delta$ is the finite difference laplacian on $\mathbb{Z}$ whose matrix elements satisfy

$$
\Delta_{i j}= \begin{cases}1 & \text { for }|i-j|=1 \\ 0 & \text { otherwise }\end{cases}
$$


The potential $V=V(\omega)$ has matrix elements

$$
V_{i j}(\omega)=\delta_{i j} v_{j}(\omega),
$$

where $v_{j}$ is the potential given in (1). Finally if we define

$$
H=-\Delta+V \text {, }
$$

we may re-write Eq. (3) as

$$
H \psi=E \psi .
$$

We will often need to restrict $H$ to intervals $J \subset \mathbb{Z}$. We do this by defining the matrix elements of the restricted operator $H^{J}$ to be

$$
H_{i, j}^{J}=H_{i, j} \text { for } i, j \in J .
$$

We note that the superscript $J$ refers to the interval on which the full Hamiltonian $H$ is restricted and that the subscripts $i, j \in \mathbb{Z}$ refer to the individual matrix elements of $H^{J}$. We will also need to decompose $H^{J}$ into Hamiltonians on sub-intervals of $J$. The reason for doing this is to allow us to use information on sub-intervals to gain information on much larger intervals and ultimately on $\mathbb{Z}$ itself. To make these ideas concrete suppose that we have the operator $H^{[a, b]}$ and $c \in(a, b)$. We wish to decompose $H^{[a, b]}$ and compare it to the operators $H^{[a, c]}$ and $H^{[c+1, b]}$. To do this we define $\Gamma$ to be the (boundary) operator which makes the following equation hold true:

$$
H^{[a, b]}=H^{[a, c]} \oplus H^{[c+1, b]}-\Gamma .
$$

We immediately note that the matrix elements of $\Gamma$ are

$$
\begin{aligned}
\Gamma_{c, c+1} & =\Gamma_{c+1, c}=1, \\
\Gamma_{i j} & =0 \quad \text { otherwise } .
\end{aligned}
$$

Now we define the corresponding Greens functions

$$
\begin{aligned}
G^{[a, b]} & \equiv\left(H^{[a, b]}-\mathscr{E}\right)^{-1}, \\
G^{[a, c]} & \equiv\left(H^{[a, c]}-\mathscr{E}\right)^{-1}, \\
G^{[c+1, b]} & \equiv\left(H^{[c+1, b]}-\mathscr{E}\right)^{-1}, \\
G^{\Gamma} & \equiv G^{[a, c]} \oplus G^{[c+1, b]} .
\end{aligned}
$$

With these definitions a simple rearrangement of Eq. (4) yields the resolvent identity

$$
G^{[a, b]}=G^{\Gamma}+G^{\Gamma} \Gamma G^{[a, b]} .
$$

We note that if $i<c<j$, then it follows (from the definition of $G^{\Gamma}$ ) that $G_{i, j}^{\Gamma}=0$. Therefore in this case Eq. (5) becomes

$$
G_{i, j}^{[a, b]}=G_{i, c}^{\Gamma} \Gamma_{c, c+1} G_{c+1, j}^{[a, b]}=G_{i, c}^{[a, c]} G_{c+1, j}^{[a, b]}
$$

for $i<c<j$. This form of the resolvent identity will be the main tool used in the proof of our result.

Outline of the Proof. Since the system (1) is ergodic, the Lyapunov exponent $\gamma(E)$ is independent of $\omega$ for almost every $\omega$. Therefore everything we prove is valid for almost every $\omega$. 
To estimate the Lyapunov exponent for (1) we will need to move $E$ slightly off the real axis, estimate the Lyapunov exponent for the complexified system and then finally relate it to the Lyapunov exponent for the original system (1). The reason for making $E$ complex is to eliminate the possibility of extremely small divisors in the Greens function $(H-E)^{-1}$.

With this in mind we modify Eq. (1) and study the system

$$
-\left(\psi_{j+1}+\psi_{j-1}\right)+\left(v_{j}-\mathscr{E}\right) \psi_{j}=0
$$

where $\mathscr{E}=E+i \delta$. Our aim in the proof which we give in Sect. III is to show that for $\delta \cong e^{-l \beta / 6}$ the Lyapunov exponent $\gamma(E+i \delta)$ of system (7) is positive and that the Lyapunov exponent $\gamma(E)$ of system (1) is "close" to $\gamma(E+i \delta)$, except for those values of $E$ which lie in a set $\Lambda$ of Lebesgue measure $\cong \delta$. Therefore $\gamma(E)$ will be positive on the complement of $\Lambda$. These ideas are made more precise in the two lemmas which follow.

Lemma 1. Suppose that $\gamma(E+i \delta)$ and $\gamma(E)$ are the Lyapunov exponents of systems (7) and (1) respectively. Then

$$
\gamma(E+i \delta)-\gamma(E)=(1 / 2) \int \ln \left[1+\delta^{2} /(E-x)^{2}\right] d \rho(x),
$$

where $d \rho$ is the density of states measure.

Proof. From the Thouless formula [9] we have

$$
\begin{aligned}
\gamma(E+i \delta)-\gamma(E) & =\int \log |E+i \delta-x| d \rho(x)-\int \log |E-x| d \rho(x) \\
& =\int \log |(E-x+i \delta) /(E-x)| d \rho(x) \\
& =\int \log |1+i \delta /(E-x)| d \rho(x) \\
& =(1 / 2) \int \log \left[1+\delta^{2} /(E-x)^{2}\right] d \rho(x) .
\end{aligned}
$$

Lemma 2. Suppose that $v$ is a positive constant. Let

$$
\Lambda=(E:|\gamma(E+i \delta)-\gamma(E)| \geqq v\} .
$$

Then meas $(\Lambda) \leqq(\pi / v) \delta$, where meas $=$ Lebesgue measure.

Proof. By Lemma 1 it is easy to see that

$$
\begin{aligned}
v \text { meas }(\Lambda) & \leqq \int_{\Lambda}[\gamma(E+i \delta)-\gamma(E)] d E \\
& =\int_{\Lambda} \int_{1}(1 / 2) \log \left[1+\delta^{2} /(E-x)^{2}\right] d \rho(x) d E \\
& =\iint_{\Lambda}(1 / 2) \log \left[1+\delta^{2} /(E-x)^{2}\right] d E d \rho(x) .
\end{aligned}
$$

Now we fix $x$ and estimate the inside integral,

$$
\int_{\Lambda} \log \left[1+\delta^{2} /(E-x)^{2}\right] d E \leqq \int_{\mathbb{R}} \log \left[1+\delta^{2} /(E-x)^{2}\right] d E \leqq 2 \pi \delta .
$$

Therefore since $\int d \rho(x)=1$ we see that meas $(\Lambda) \leqq(\pi / v) \delta$.

We now briefly discuss why the Lyapunov exponent of the modified system (7) is positive. The technical proof will be given in Sect. III. 
Suppose that $\omega$ and $E$ are fixed. Without loss of generality we may assume that the following picture holds true with $J_{1}$ being the first interval in $\left\{J_{n}\right\}_{n=-\infty}^{\infty}$ whose center $a_{1}$ is a non-negative integer.

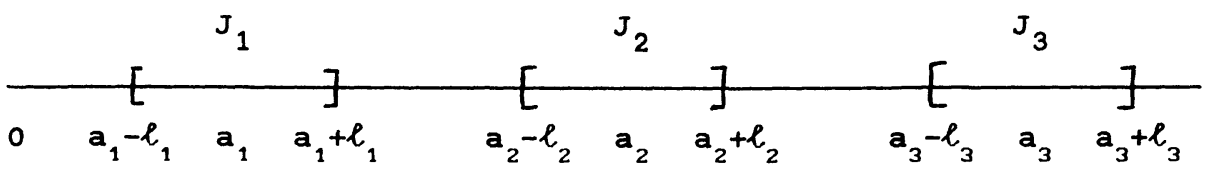

Fig. 1

We will prove that in every interval $J_{n}=\left[a_{n}-l_{n}, a_{n}+l_{n}\right)$ the Greens function $G^{J_{n}}=\left(H^{J_{n}}-\mathscr{E}\right)^{-1}$ decays exponentially fast. In particular we will show that

$$
\left|G_{a_{n}, a_{n}+l_{n}}^{J_{n}}\right| \leqq C e^{-\beta l_{n}} \leqq C e^{-\beta l},
$$

where $C$ and $\beta$ are positive constants. (See Sect. III, Lemma 3). We note that in the remaining intervals $I \subset \mathbb{Z}$ we always have the crude bound

$$
\left\|G^{I}\right\|=\left\|\left(H^{I}-\mathscr{E}\right)^{-1}\right\|=\left\|\left(H^{I}-E-i \delta\right)^{-1}\right\| \leqq 1 / \delta .
$$

If we define $\delta \cong e^{-\beta l / 6}$ we see that

$$
\left\|G^{I}\right\| \leqq \text { Const } \cdot e^{\beta l / 6} .
$$

Finally we will use estimates (9), (11) and the resolvent identity (5) to prove that the full Greens function $G=(H-\mathscr{E})^{-1}=(H-E-i \delta)^{-1}$ decays exponentially fast. More specifically (see Sect. III, Lemma 4) we will prove that

$$
\begin{aligned}
\left|G_{0, a_{n}}\right| & \leqq e^{-(\beta L / 4) n} \\
& =e^{-(\beta L / 4 L)(n L)},
\end{aligned}
$$

where $\beta$ is the constant defined in Eq. (9). By assumption (iii) and Fig. 1 it follows that $a_{n} \leqq n L$, therefore

$$
\left|G_{0, a_{n}}\right| \leqq e^{-(\beta l / 4 L) a_{n}} .
$$

Since the Lyapunov exponent is related to the rate of decay of the Greens function it follows from (12) that

$$
\gamma(E+i \delta) \geqq \beta l / 4 L .
$$

Finally by Lemma 2 with $v=\beta l / 8 L$ we see that

$$
|\gamma(E+i \delta)-\gamma(E)| \leqq \beta l / 8 L,
$$

except for those values of $E$ which belong to a set $\Lambda$ which has Lebesgue measure $\cong \delta=$ Const $\cdot e^{-\beta l / 6}$. By combining estimates (13) and (14) we find that $\gamma(E) \geqq \beta l / 8 L$ on the complement of $\Lambda$.

With the notation and sketch of the proof behind us we will now state and prove our main result. 


\section{Proof of the Main Theorem}

The proof of our theorem rests upon the following two lemmas.

Lemma 3. Let $G^{J_{n}}=\left(H^{J_{n}}-\mathscr{E}\right)^{-1}$, where $\mathscr{E}=E+i \delta$. Then

$$
\left|G_{i, j}^{J_{n}}\right| \leqq C e^{-\beta|i-j|},
$$

where $C=1 /(\zeta-2)$ and $\beta=\log (\zeta / 2)$.

Proof. By condition (iv) we have

$$
\left|v_{j}-\mathscr{E}\right|=\left|v_{j}-E-i \delta\right| \geqq\left|v_{j}-E\right| \geqq \zeta>2
$$

for $j \in J_{n}$. We expand $G^{J_{n}}=\left(H^{J_{n}}-\mathscr{E}\right)^{-1}=\left(-\Delta^{J_{n}}+V^{J_{n}}-\mathscr{E}\right)^{-1}$ in powers of $\zeta^{-1} \Delta^{J_{n}}$ to get

$$
\begin{aligned}
\left|G_{i, j}^{J_{n}}\right| & \leqq\left|\zeta^{-1} \sum_{k \geqq|i-j|}\left(\zeta^{-1} \Delta^{J_{n}}\right)_{i, j}^{k}\right| \\
& \leqq \zeta^{-1} \sum_{k \geqq|i-j|}(2 / \zeta)^{k} \equiv C e^{-\beta|i-j|},
\end{aligned}
$$

where $C=1 /(\zeta-2)$ and $\beta=\log (\zeta / 2)$.

Lemma 4. Let $G=(H-\mathscr{E})^{-1}=(H-E-i \delta)^{-1}$ and let $\delta=(2 C)^{1 / 3} e^{-\beta l / 6}$, where $\beta$ and $C$ are the constants defined in Lemma 3. Suppose that the intervals $J_{n}$ are as in Fig. 1. Then

$$
\left|G_{0, a_{n}}\right| \leqq e^{-(\beta l / 4 L) a_{n}} .
$$

Proof. We apply (6) twice to $G=G^{(-\infty, \infty)}$ to get

$$
G_{a_{1}, a_{n}}=G_{a_{1}, a_{1}+l_{1}}^{\left(-\infty, a_{1}+l_{1}\right]} G_{a_{1}+l_{1}+1, a_{2}-1}^{\left(-\infty, a_{2}-1\right]} G_{a_{2}, a_{n}},
$$

which upon iteration yields

$$
G_{a_{1}, a_{n}}=\left(\prod_{k=1}^{n-1} G_{a_{k}, a_{k}+l_{k}}^{\left(-\infty, a_{k}+l_{k}\right]}\right)\left(\prod_{k=1}^{n-1} G_{a_{k}+l_{k}+1, a_{k+1}-1}^{\left(-\infty, a_{k+1}-1\right]}\right) G_{a_{n}, a_{n}} .
$$

Another application of (6) to $G=G^{(-\infty, \infty)}$ gives us

$$
G_{0, a_{n}}=G_{0, a_{1}-1}^{\left(-\infty, a_{1}-1\right]} G_{a_{1}, a_{n}} .
$$

We will use (17) and (18) to get the desired bound on $G_{0, a_{n}}$. To estimate the first product in (17) we will use Lemma 3 to show that

$$
\left|G_{a_{k}, a_{k}+l_{k}}^{\left(-\infty, a_{k}+l_{k} \mathrm{~J}\right.}\right| \leqq 2 C e^{-\beta l} / \delta .
$$

Every other term in Eqs. (17) and (18) is trivially bounded by $(1 / \delta)$. For the moment we assume that (19) is true and combine it with (17), (18) and the definition of $\delta$ to get

$$
\begin{aligned}
\left|G_{0, a_{n}}\right| & \leqq(1 / \delta)\left(2 C e^{-\beta l} / \delta\right)^{n-1}(1 / \delta)^{n-1}(1 / \delta) \\
& \leqq\left(2 C e^{-\beta l} / \delta^{3}\right)^{n-1} \\
& =e^{-\beta l(n-1) / 2} \\
& \leqq e^{-(\beta l / 4 L) n L}
\end{aligned}
$$


By assumption (iii) and Fig. 1 we see that $a_{n} \leqq n L$ which implies that

$$
\left|G_{0, a_{n}}\right| \leqq e^{-(\beta L / 4 L) a_{n}} \text {. }
$$

It now remains to prove (19). We use (6) again to write

$$
G^{\left(-\infty, a_{k}+l_{k}\right]}=G^{\Gamma}+G^{\Gamma} \Gamma G^{\left(-\infty, a_{k}+l_{k}\right]},
$$

where

$$
\begin{aligned}
G^{\Gamma} & =G^{\left(-\infty, a_{k}-l_{k}-1\right]} \oplus G^{\left[a_{k}-l_{k}, a_{k}+l_{k}\right]} \\
& =G^{\left(-\infty, a_{k}-l_{k}-1\right]} \oplus G^{J_{k}}
\end{aligned}
$$

Now by (21) and (22) we get

$$
\mid G_{a_{k}, a_{k}+l_{k}}^{\left(-\infty, a_{k}+l_{k}\right]}=G_{a_{k}, a_{k}+l_{k}}^{J_{k}}+G_{a_{k}, a_{k}-l_{k}}^{J_{k}} G_{a_{k}-l_{k}-1, a_{k}+l_{k}}^{\left(-\infty, a_{k}+l_{k}\right]} .
$$

We finally use Lemma 3, assumption (ii) and Eq. (10) to obtain (19),

$$
\begin{aligned}
\left|G_{a_{k}, a_{k}+l_{k}}^{\left(-\infty, a_{k}+l_{k}\right]}\right| & \leqq C e^{-\beta l_{k}}+C e^{-\beta l_{k}}(1 / \delta) \\
& \leqq 2 C e^{-\beta l_{k}} / \delta \\
& \leqq 2 C e^{-\beta l} / \delta .
\end{aligned}
$$

Remark. In the preceding proof we needed to use the fact that $\delta<1$. This is an explicit requirement on the largeness of $l$. In particular we see that if

$$
l>2 \log [2 /(\zeta-2)] / \log (\zeta / 2),
$$

then it follows from the definition of $C$ that $\delta<1$.

We can now prove our main result:

Theorem. For system (1) with conditions (i-iv) there exists positive constants $\beta$ and $B$ such that

$$
\operatorname{meas}\{E: \gamma(E)=0\} \leqq B e^{-\beta L / 6} .
$$

The constant $\beta$ depends on $\zeta$ alone and $B$ depends on $\zeta$ and on the ratio $l / L$.

Remark. The proof we give actually yields the more general result

$$
\text { meas }\{E: \gamma(E) \leqq \beta l / 8 L\} \leqq B e^{-\beta l / 6} \text {. }
$$

Proof. From Lemma 4 we see that $\gamma(E+i \delta) \geqq \beta l / 4 L$, provided that we choose $\delta=(2 C)^{1 / 3} e^{-\beta l / 6}$. Now Lemma 2 implies that

$$
\text { meas }\{E:|\gamma(E+i \delta)-\gamma(E)| \geqq \beta l / 8 L\} \leqq(8 \pi L / \beta l) \delta \equiv B e^{-\beta l / 6} .
$$

Therefore

$$
\begin{aligned}
\operatorname{meas}\{E: \gamma(E)=0\} & \leqq \operatorname{meas}\{E: \gamma(E) \leqq \beta l / 8 L\} \\
& \leqq \operatorname{meas}\{E:|\gamma(E+i \delta)-\gamma(E)| \geqq \beta l / 8 L\} \\
& \leqq B e^{-\beta l / 6}
\end{aligned}
$$

Acknowledgements. I would like to thank Professor T. Spencer for suggesting this work to me and for the many useful conversations leading to its completion. I would also like to thank Y. Last for reading this paper and for making several useful corrections and suggestions. 


\section{References}

1. Spencer T.: Ergodic Schrödinger Operators. Analysis, Et Cetera. New York: Academic Press, 1990

2. Herman, M.: Une methode pour minorer les exposants de Lyapunov et quelques exemples montrant le caractere local d'un theoreme d'Arnold et de Moser sur let tore en dimension 2. Commun. Math. Helv. 58, 453-502 (1983)

3. Sorets, E., Spencer, T.: Positive Lyapunov Exponents for Schrödinger Operators with QuasiPeriodic potentials. Commun. Math. Phys. 142, 543-566 (1991)

4. Spencer, T.: Localization for random and Quasi-Periodic potentials. J. Stat. Phys. 51, 10091019 (1988)

5. Surace, S.: The Schrödinger Equation with a Quasi-Periodic potential. Transactions of AMS. Vol 320, Number 1, 321-369 (1990)

6. Dinaburg, E.I., Sinai, Ya.G.: One dimensional Schrödinger equation with a Quasi-Periodic potential. Funct. Anal. Appl. 9, 279-289 (1975)

7. Sinai, Ya.G.: Anderson Localization for one dimensional difference Schrödinger operators with a Quasi-Periodic potential. J. Stat. Phys. 46, 861-909 (1987)

8. Frohlich, J., Spencer, T., Wittwer, P.: Localization for a class of one dimensional QuasiPeriodic potentials. Commun. Math. Phys. 132, 5-25 (1990)

9. Cycon, H., Froese, R., Kirsch, W., Simon, B.: Schrödinger Operators. Berlin, Heidelberg, New York: Springer, 1987

Communicated by B. Simon 
